Bot. Helv. 115 (2005): i-v

0253-1453/05/01000i-5

DOI 10.1007/s00035-005-0008-4

(C) Birkhäuser Verlag, Basel, 2005

Botanica Helvetica

Notiz der Redaktorin

\title{
Botanica Helvetica: Entwicklungen 1981-2004 und in der Zukunft
}

Mit ihrer 115-jährigen Publikationsgeschichte gehört Botanica Helvetica zu den traditionsreichsten botanischen Fachzeitschriften der Welt. Dass sie noch immer eine lebendige Zeitschrift ist, konnte ich seit meinem Einstieg als Redaktorin im Herbst 2004 direkt erfahren: an der hohen Zahl der eingegangenen Manuskripte; an der spontanen Bereitschaft vieler Gutachter, die Eingänge zu beurteilen und zu verbessern; an der vielseitigen Zusammensetzung der neuen Redaktionskommission (siehe Impressum); an den Reaktionen von Kollegen, Lesern und Leserinnen - von Glückwünschen über Veränderungswünsche bis zur Frage, ob das nicht sehr viel Arbeit gibt. Es freut mich, die Redaktion einer Zeitschrift übernehmen zu dürfen, die in der Schweiz so gut etabliert ist. Andererseits bedeutet es auch eine Herausforderung: Wird wohl beim nächsten Redaktionswechsel Botanica Helvetica noch immer soviel Unterstützung erfahren? Was braucht es, damit die Zeitschrift angesichts der veränderten Rahmenbedingungen für LeserInnen interessant und für AutorInnen attraktiv bleibt?

Sehr hilfreich bei diesen Überlegungen waren Aufzeichnungen zur Entwicklung der Inhalte, der Autorenschaft, des Umfangs und der Publikationskosten von Botanica Helvetica, welche die Redaktoren Herr Prof. H.R. Hohl (1981-1995) und Herr Prof. H.P. Ruffner (1996-2004) seit 1981 systematisch geführt haben. Deshalb möchte ich hier einige Entwicklungen aufzeigen und auf dieser Grundlage unsere Zukunftspläne erläutern.

\section{Zusammensetzung der Beiträge}

Die Diagramme in den Abbildungen 1 und 2 zeigen, wie sich die Zusammensetzung der in Botanica Helvetica publizierten Artikel seit 1981 entwickelt hat. Hierzu wurden jeweils die Artikel von sechs Bänden zusammengefasst.

Die Zusammensetzung der Autorenschaft bezüglich ihrer geographischen Herkunft (Adresse der Erstautoren) ist praktisch konstant geblieben: gut die Hälfte der Beiträge stammen aus der Deutschschweiz, 16-20\% aus der Romandie und 26-30\% aus dem Ausland (Abb. 1a). Etwas anders sehen die Zahlen zur geographischen Lage der Untersuchungen aus: in den achtziger Jahren untersuchten viele Schweizer Autoren die Flora anderer Regionen (vor allem im Mittelmeerraum); in jüngster Zeit liegen die Untersuchungsobjekte primär in der Schweiz. Das vorliegende Heft folgt dem Trend: $75 \%$ der ErstautorInnen arbeiten an einem Schweizer Institut, 88\% der Untersuchungsobjekte lagen in der Schweiz.

Die meisten Autoren von Botanica Helvetica arbeiten an Hochschulinstituten. Beiträge aus land- und forstwirtschaftlichen Forschungsanstalten, Fachstellen, Planungsbüros oder Privatadressen haben aber zugenommen: von 13\% in der Periode 1981-1986 auf $32 \%$ in der Periode 1999-2004, vor allem dank Beiträgen der Eidg. Forschungsanstalt WSL und der Stiftung ZDSF. 
(a)

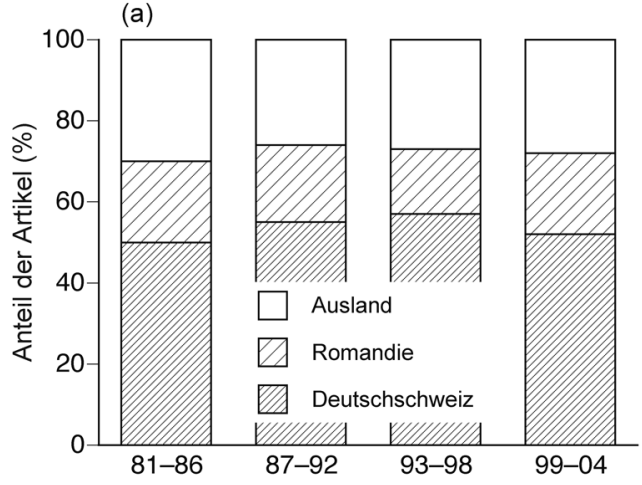

(b)

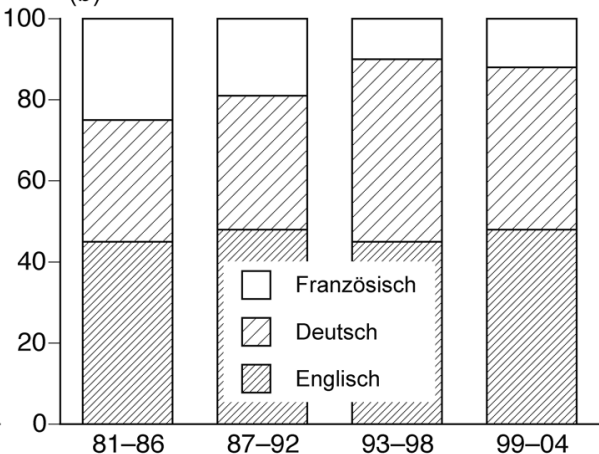

Abb. 1. Zusammensetzung der Artikel von Botanica Helvetica bezüglich (a) der geographischen Herkunft der Autoren und (b) der Sprache, für vier Perioden von sechs Jahren. Daten zusammengestellt durch H.R. Hohl und H.P. Ruffner.

Der Anteil englischsprachiger Artikel ist konstant geblieben. Deutschsprachige Artikel wurden häufiger, französischsprachige seltener (Abb. 1b). Italienisch blieb eine Ausnahme. Auch bezüglich der Sprache folgt das vorliegende Heft (50\% Englisch, 38\% Deutsch, 12\% Französisch) dem Durchschnitt der letzten sechs Bände. Alle englischsprachigen Artikel enthalten eine Zusammenfassung in einer Landessprache.

Der Anteil von Artikeln zu Systematik, Vegetationskunde und Ökologie hat seit 1981 zugenommen (Abb. 2a). Bei den Gebieten Morphologie, Zytologie und Genetik besteht insgesamt kein klarer Trend. Physiologische, biochemische, mykologische und phytopathologische Arbeiten, die 1981-1986 gemeinsam ein gutes Drittel der Beiträge ausmachten, sind fast vollständig aus Botanica Helvetica verschwunden (Abb. 2a). Dies kann sich auch wieder ändern: Manuskripte aus dem Gesamtgebiet der Botanik und der Mykologie sind weiterhin bei uns willkommen und werden durch Experten des betreffenden Fachgebiets begutachtet.

Der Anwendungsbezug hat zugenommen: in den achtziger Jahren befassten sich nur $7 \%$ der Beiträge mit Naturschutz und Umweltveränderungen; in der Periode 1999-2004 waren es 25\% (Abb. 2b). Das vorliegende Heft enthält gar vier Beiträge zu seltenen und gefährdeten Pflanzenarten und drei Beiträge zu invasiven Pflanzen. Auch der traditionelle Beitrag aus der Reihe der „Fortschritte“ analysiert die floristischen Neufunde in Bezug auf die Roten Listen.

\section{Wissenschaftliche Bedeutung}

Seit 1997 veröffentlicht das Institute of Scientific Information, wie oft Artikel einer Zeitschrift durchschnittlich in den zwei ersten Jahren nach deren Publikation in anderen Artikeln zitiert werden. Der Impact Factor von Botanica Helvetica lag bis $2001 \mathrm{im}$ Bereich 0.6-0.7 (Abb. 3) und somit im Mittelfeld der botanischen Zeitschriften. Den Höhepunkt bildete das Jahr 1999 mit einem Impact Factor von 0.81. Seitdem nahm der 

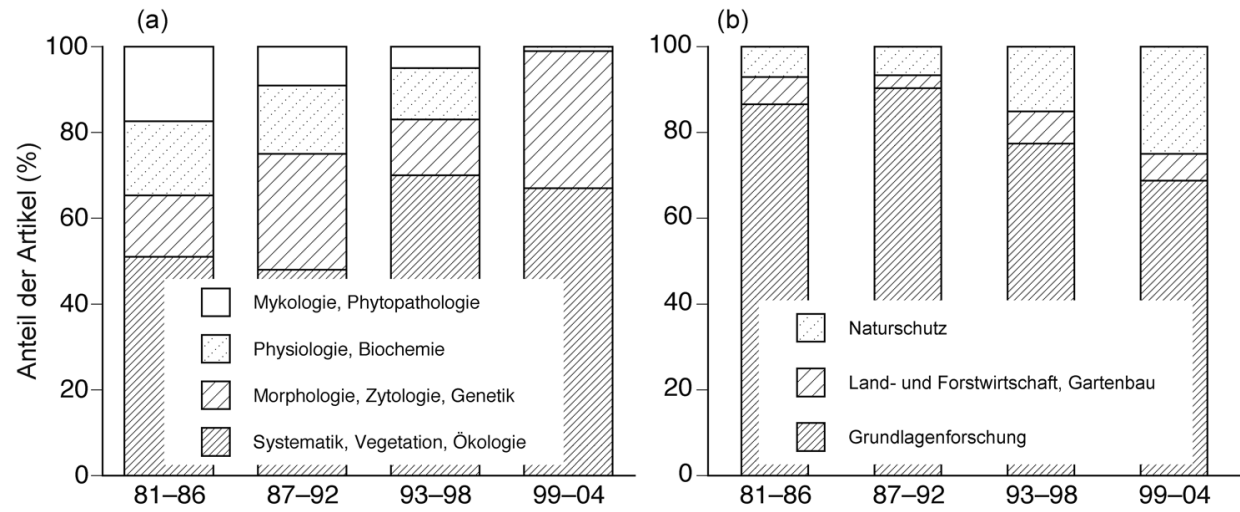

Abb. 2. Zusammensetzung der Artikel von Botanica Helvetica bezüglich (a) des Fachgebiets und (b) dem Anwendungsbereich für vier Perioden von sechs Jahren. Daten zu (a) zusammengestellt durch H.R. Hohl und H.P. Ruffner.

Impact unserer Zeitschrift stetig ab (Abb. 3). Problematisch ist dies, weil es für die Karriere von AkademikerInnen und die Bewertung ihrer Institute zunehmend wichtig ist, welchen Impact Factor die Zeitschriften haben, in denen sie publizieren. Damit Botanica Helvetica ein attraktives Publikationsorgan für Forschende an Schweizer Hochschulen bleibt, muss ihr Impact wieder zunehmen.

Warum ist der Impact Factor gesunken? Vermutlich hat nicht die Qualität oder Relevanz der Beiträge abgenommen, sondern deren Verbreitung. Seit 1997 haben die meisten anderen botanischen Zeitschriten eine elektronische Version auf dem Internet zugänglich gemacht. Wenige Mausklicks genügen, um einen Artikel herunterzuladen und auszudrucken. Anders Botanica Helvetica: Die Beiträge werden zwar in den wichtigsten bibliographischen Datenbanken erfasst, die Artikel selbst sind aber nicht in elektronischer Form zugänglich. Noch im Jahr 2000 kam der damalige SBG-Vorstand (dem ich als Sekretärin angehörte) zusammen mit dem Birkhäuser-Verlag zu dem Schluss, dass kein dringender Bedarf für eine elektronische Version besteht; wenige Jahre später ist sie einfach unverzichtbar geworden.

\section{Elektronische Publikation}

$\mathrm{Ab}$ diesem Band ist Botanica Helvetica in elektronischer Form auf dem Internet verfügbar; die Artikel sind direkt mit wichtigen Datenbanken und Suchdiensten (wie das Web of Science, Biosis Previews oder Springer-Link) verbunden. Dies allein dürfte den Impact der Zeitschrift steigern; es eröffnet auch neue Möglichkeiten, die Verbreitung und Attraktivität der Beiträge sowie deren Wahrnehmung durch ausländische Leser zu erhöhen.

- Die Autoren erhalten von ihren Artikeln künftig eine PDF-Datei, welche sie via EMail in wenigen Minuten und ohne Unkosten an Kollegen senden können; sie können den Artikel auch auf eine persönliche Homepage setzen. 


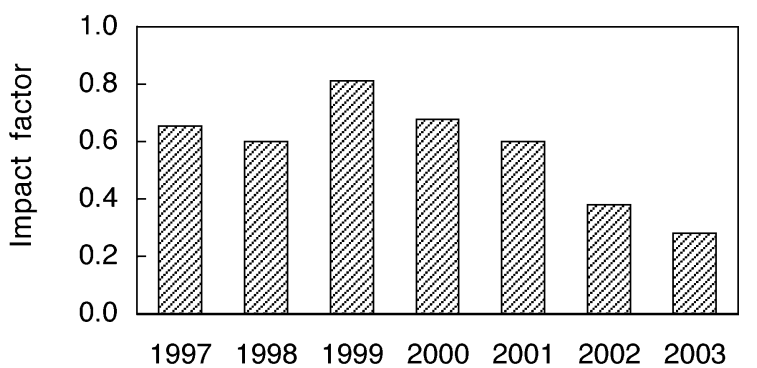

Abb. 3. Entwicklung des wissenschaftlichen „Impacts“ von Botanica Helvetica. Daten vom Science Citation Index (http://wos.consortium.ch).

- Eine englischsprachige Kurzversion von Beiträgen in Landessprachen kann auf dem Internet verfügbar sein, so dass Kollegen im Ausland zumindest die wichtigsten Inhalte eines Artikels schnell und ohne sprachliche Schwierigkeiten lesen können. Aus dem vorliegenden Heft ist der Beitrag von P. Vittoz et al. in dieser Form erhältlich.

- Abbildungen (Fotos oder Karten) können ohne Mehrkosten in der elektronischen Version farbig erscheinen, selbst wenn sie weiterhin in Graustufen gedruckt werden.

- Elektronische Anhänge können bei umfangreichen Tabellen zahlreiche Druckseiten oder teure Faltblätter ersetzen; eventuell können sie auch direkt von den Lesern übernommen und für weitere Auswertungen genutzt werden.

Über den Gebrauch dieser Optionen entscheiden primär die Autoren selbst; die Redaktion wird sie dabei aber gerne unterstützen. Die gedruckten Hefte erscheinen weiterhin zweimal pro Jahr und werden wie bisher allen Mitgliedern der SBG per Post zugestellt.

\section{Zukunftspläne}

Die vielseitige Unterstützung von Botanica Helvetica innerhalb der Schweiz zeigt, dass die heutige thematische Zusammensetzung der Artikel und deren sprachliche Vielfalt den Bedürfnissen unserer Leserschaft entsprechen. Dies ist vor allem der sorgfältigen Auswahl der Beiträge durch den bisherigen Redaktor, Herr Prof. H.P. Ruffner, zu verdanken: wichtigstes Kriterium war immer die wissenschaftliche und sprachliche Qualität; von der Relevanz für Schweizer LeserInnen hing aber ab, inwiefern ungenügende Manuskripte überarbeitet und wieder eingereicht werden durften. Dieses Auswahlprinzip möchte ich beibehalten.

Für die formale Qualität der Beiträge sorgt als stellvertretende Redaktorin Frau Dr. D. Nierhaus-Wunderwald, die seit 2002 (ebenso wie ihr Vorgänger, Herr Dr. G. Nogler) jeden Beitrag sehr genau überprüft, Inkonsistenzen bereinigt, und die Druckfahnen und den Umbruch kontrolliert. Herzlichen Dank, Dagmar!

Der Birkhäuser-Verlag hat die Produktion seit Jahren zuverlässig und entgegenkommend betreut. Ich freue mich auf die Fortsetzung dieser guten Zusammenarbeit. 
Unverzichtbar ist hierfür die finanzielle Unterstützung durch die Schweizerische Akademie der Naturwissenschaften (ScNat), welche die Druckkosten teilweise übernimmt. Wir hoffen, auch in Zukunft mit dieser Unterstützung rechnen zu dürfen.

Mit diesem Band erhält Botanica Helvetica einen neuen Umschlag. Das zentrale Motiv deutet den heutigen thematischen Schwerpunkt der Zeitschrift an; zugleich signalisieren grosse blaue Bereiche, dass neben dem ,grünen“ Schwerpunkt Beiträge aus allen Gebieten der Botanik willkommen sind. Die sprachliche Vielfalt ist auf der Rückseite dargestellt. Wir planen übrigens, das zentrale Motiv regelmässig auszuwechseln und werden Sie, liebe Leserinnen und Leser, zu gegebener Zeit um Vorschläge bitten.

Als weitere Neuerung sind AutorInnen eingeladen, sich am Ende ihres Beitrags ganz kurz der Leserschaft vorzustellen (siehe unten). Für viele LeserInnen ist diese Information interessant, für die Autoren bleibt sie aber freiwillig.

Schliesslich möchten wir in Zukunft mit einer besonders gekennzeichneten Sektion „Botanische Notizen“ Raum für Kurzbeiträge (1-4 Seiten) bieten, welche nicht den Anspruch eines vollen wissenschaftlichen Artikels erheben, und für deren Annahme einfacherere Bedingungen gelten (in der Regel keine externe Begutachtung). In Frage kommen kleine botanische und vegetationskundliche Feldstudien, Kurzfassungen von Diplomarbeiten oder erste Resultate von Langzeitstudien. Buchbesprechungen sind möglich, sofern sie die Flora oder Vegetation der Schweiz betreffen. Einfache botanische Fundmeldungen sollten weiterhin dem ZDSF gemeldet werden (vgl. S. 83 in diesem Heft) und erscheinen wie bisher in den „Fortschritten“. Die neue Sektion ersetzt das bisherige „Bulletin Vegetatio Helvetica“ (Mitteilungen der Schweizerischen Gesellschaft für Vegetationsforschung); wir hoffen, dadurch mehr Beiträge von BotanikerInnen zu veröffentlichen, die nicht an Hochschulinstituten arbeiten.

$\mathrm{Ob}$ sich diese Neuerungen bewähren, entscheiden Sie, liebe Leserinnen und Leser, mit ihrem Interesse an der Zeitschrift, mit ihren Rückmeldungen, und vor allem mit den Manuskripten, die Sie als Autorinnen und Autoren bei uns einreichen. Ich freue mich, demnächst von Ihnen zu hören oder zu lesen!

Sabine Güsewell

Sabine Güsewell hat an der ETH Zürich Umweltnaturwissenschaften studiert und 1997 zur Verschilfung von Feuchtwiesen promoviert. Nach einem Projekt zur Nährstoffökologie von Feuchtgebieten in den Niederlanden unterrichtet sie am Geobotanischen Institut der ETH Zürich Pflanzenökologie und Vegetationskunde. In ihrer Forschung interessiert sie sich besonders dafür, wie ausdauernde Krautpflanzen ihre Nährstoffnutzung an Standort und Bewirtschaftung anpassen und wie sie auf ihren Standort einwirken.

(D) To access this journal online:

(4P) http://www.birkhauser.ch 
00 Editorial 24.5.2005 9:48 Uhr Seite vi

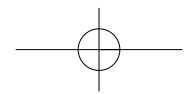

